\title{
OGEO: SISTEMA DE NAVEGACIÓN INTERIOR PARA LA ORIENTACIÓN Y MOVILIDAD DE PERSONAS CON DISCAPACIDAD VISUAL
}

OGEO: INDOOR NAVIGATION SYSTEM FOR ORIENTATION AND MOBILITY OF THE VISUALLY IMPAIRED PEOPLE

\section{Manuel Gomez ${ }^{1}$, Víctor Rodríguez ${ }^{2}$, Rafael Vejarano ${ }^{3}$ y Hector Montes ${ }^{4}$}

Fecha de envío: 8 de mayo de 2020

Fecha de aceptación: 24 de mayo de 2020.

\section{Resumen}

El movilizarse libremente implica, para las personas con discapacidad visual, adquirir técnicas de orientación y movilidad que les permita ejercer su autonomía e independencia. La orientación le permite reconocer su posición con respecto al entorno, mientras que la movilidad le permite moverse de manera segura y eficiente de un lugar a otro. Sin embargo, el recorrer un lugar desconocido es un reto para una persona con discapacidad visual (PcDV). En este trabajo de investigación y desarrollo se construye OGeo, un sistema de orientación y navegación para interiores de edificios que mediante el uso de balizas (dispositivos iBeacon) y celulares basados en Android permite que una PcDV pueda desplazarse en el interior de un edificio. El sistema está basado en instrucciones de voz, que guiarán a la $\mathrm{PcDV}$ de un punto $\mathrm{A}$ hasta

\footnotetext{
${ }^{1}$ Estudiante de Licenciatura en Desarrollo de Software. Centro Regional de Coclé, Universidad Tecnológica de Panamá. manuel.gomez2@utp.ac.pa

2 Estudiante de Licenciatura de Desarrollo de Software. Centro Regional de Coclé, Universidad Tecnológica de Panamá.victor.rodriguez@utp.ac.pa

3 Magíster en Desarrollo de Software. Centro Regional de Coclé, Universidad Tecnológica de Panamá, rafael.vejarano@utp.ac.pa

4 Doctor en Arquitectura de Computadores y Automática. Facultad de Ingeniería Eléctrica. Universidad Tecnológica de Panamá. Centro de Automática y Robótica (CSIC-UPM), España, hector.montes1@utp.ac.pa
} 
OGEO: Sistema de navegación interior para la orientación y movilidad de personas con discapacidad visual

un punto $\mathrm{B}$, con instrucciones precisas, paso a paso. El objetivo fundamental, desde un punto de vista social, es el de aportar ayudas en la inclusión social de las PcDV, en donde es evidente que proporcionará una mayor inserción escolar.

Palabras clave: Persona con discapacidad visual, PcDV, baliza, iBeacon, sistema de navegación en interiores.

\section{Abstract}

Moving freely means, for visually impaired people, acquiring spatial orientation and mobility techniques that allow them to achieve their autonomy and independence. Orientation allows them to recognize their position according to the environment, while mobility allows them to move safely and efficiently from one place to another. However, navigating around an unknown place is a challenge for visually impaired people (ViP). In this research and development work, OGeo is created, an orientation and navigation system for interiors of buildings that uses markers (iBeacon devices) and mobile phones based on Android. This system allows a ViP to move inside a building independently. The system is based on voice instructions, which will guide a ViP from point $A$ to point $B$, using precise instructions, step by step. The fundamental objective, from a social point of view, is to provide support for the social inclusion of ViP, where it is evident that it will provide greater school inclusiveness.

Key words: Visually impaired person, ViP, marker, iBeacon, indoor navigation system.

\section{INTRODUCCIÓN}

Los edificios, centros comerciales, aeropuertos, estaciones de metro, grandes lugares cerrados, entre otros, se han construido a lo largo de la historia sin tener en cuenta las necesidades específicas de las personas con discapacidad visual (PcDV). De igual forma, las barreras arquitectónicas y obstáculos o nulas/pocas señalizaciones de estos, así como la deficiencia de iluminación imposibilitan el acceso de las PcDV a estos lugares desconocidos. Sin embargo, en países europeos, concretamente en España, se están llevando a cabo iniciativas de diseño en las nuevas construcciones de edificios y en la adecuación de otros, para que tengan un diseño arquitectónico para todas las personas, con la finalidad de 
que exista una accesibilidad universal, sin barreras (López Pereda y Borau Jordán, 2011; ONCE, 2011). En la actualidad, se continúa con este tipo de políticas de inclusión social, considerando siempre mejoras en este sentido. Sin embargo, aún hay mucho trabajo por hacer y, más aún, en los países en vías de desarrollo.

Los dispositivos móviles brindan el beneficio de la portabilidad y rápido acceso a la información; sin embargo, la ausencia de teclas en sus pantallas, completamente lisas, provoca que la interacción de las PcDV con ellas sea muy compleja, e incluso nula, en muchos casos. Las interfaces no proveen distinción de controles y espacios de visualización/interacción que se puedan percibir mediante el tacto, provocando que la detección de elementos en una interfaz de usuario (IU) sea muy difícil para las PcDV. E simple trabajo de rellenar un formulario, determinar el inicio de un mensaje cuando se lee un email es una tarea tediosa para una PcDV. Las tecnologías usadas para asistir la traducción del texto visual en los Smartphone a un formato accesible para PcDV están basadas en el canal auditivo, TTS o text-to-speech, en inglés. Esta tecnología no resuelve por sí sola los problemas de accesibilidad a la información ya que, casi en su totalidad, las aplicaciones para móviles están desarrolladas para personas que pueden ver. Esto es debido a que los desarrolladores no tienen un punto de comprensión y entendimiento de como las PcDV interactúan con la tecnología, lo cual es evidente.

La autonomía para una PcDV se ve obstaculizada, ya sea porque no existen herramientas que permitan que estas personas se orienten dentro de determinados lugares por sí mismas, o porque sencillamente la cooperación de terceras personas que están a su alrededor no es suficiente para ayudarles en determinado caso. Además, en países donde existen problemas de desordenamiento vial, falta de dispositivos o carteles de avisos en lugares públicos, entre otros múltiples detalles, donde no se toma en cuenta a las personas con discapacidad visual, termina siendo un factor concluyente a la hora de intentar desenvolverse de manera independiente, ya sea en un área abierta o dentro de algún edificio. 
OGEO: Sistema de navegación interior para la orientación y movilidad de personas con discapacidad visual

El objetivo de OGeo, desde un punto vista científico o tecnológico, es desarrollar un sistema de navegación y posicionamiento, utilizando teléfonos inteligentes para la ayuda a Personas con Discapacidad Visual (PcDV) en su orientación y movilidad en entornos cerrados de forma independiente. Este sistema se desarrolla dentro del marco del proyecto MOVIDIS-II, el cual es financiado por la SENACYT con el contrato por mérito $\mathrm{N}^{\circ}$ 99-2018-4FID17-031, cuyo objetivo, desde una perspectiva social, es desarrollar sistemas basados en la TIC para favorecer la inclusión social de PcDV (Carballeda, Arcia, Pérez y Montes, 2016; Henríquez, Vejarano y Montes, 2017; Montes, et al., 2016; Montes, et al., 2018; Montes, et al., 2019). Por lo tanto, de manera más específica, la utilización de este sistema favorecerá a la educación inclusiva, ya que las PcDV, de cualquier edad, podrán contar con un sistema que les ayudará en su autonomía de movilidad para acudir a las aulas de clases y a desplazarse por el edificio de una manera más segura.

OGeo ha sido diseñado e implementado dentro del marco de una investigación basada en diseño (IBD) (De Benito y Salinas, 2016), orientado, desde el punto de vista de innovación educativa, a proporcionar ayudas a las PcDV para favorecer su educación inclusiva. Ya que se introduce, de manera indirecta, un nuevo elemento, en este caso, para que estas personas puedan asistir a los centros escolares. Por lo tanto, el sistema OGeo está orientado a resolver problemas prácticos, que adicionalmente al fin planteado anteriormente, contribuye a generar nuevos conocimientos para la movilización de PcDV en interiores de edificios, ya que no solamente se crea un producto tangible, sino también, nuevos procesos y protocolos.

El producto creado, con base en el desarrollo de software y la tecnología de la información y comunicación (TIC), ha sido desarrollado utilizando el método de prototipado (Ferré, 2005), con la finalidad de ser evaluado durante las etapas de su desarrollo. Por lo tanto, se ha realizado un desarrollo y evaluación iterativa hasta lograr un sistema que presente una eficiencia alta, desde el punto de vista usuario-sistema.

\section{AYUDAS VISUALES}

\section{ELECTRÓNICAS}

En la literatura se encuentran diversos trabajos dirigidos a presentar ayudas visuales para PcDV, desde las 
que poseen una visión parcial hasta las que padecen ceguera. Por ejemplo, existe un trabajo que presenta una pantalla granulada basada en la profundidad de las imágenes, que permiten mejorar el reconocimiento de objetos y puede ayudar a la navegación de personas con visión parcial (Hicks, et al., 2013). Por otro lado, las técnicas de visión por computadora pueden resaltar objetos tales como el piso, paredes, personas y autos; sin embargo, la demanda computacional de algoritmos para realizar este trabajo en tiempo real es muy alta y compleja (Gelgon \& Bouthemy, 2000). Las cámaras de profundidad ofrecen una manera eficiente para identificar objetos individuales en el ambiente con un consumo mínimo de recursos computacionales (Romero y Rodríguez, 2018).

Un sistema de navegación basado en análisis de imágenes (Rivera-Rubio, Arulkumaran, Rishi, Alexiou, \& Bharath, 2016), se centra en la recolección de imágenes de baja resolución captada ya sea por el Smartphone, GoogleGlass, o una cámara remota conectada al dispositivo que porta la PcDV, mediante el cual esta persona recorre un determinado espacio, del que se han obtenido imágenes en recorridos previos, para realizar las comparaciones con las imágenes actuales y las que están almacenadas en una base de datos, para ayudar en la navegación.

\section{VirtualEyes (García y} Nahapetian, 2015) es un sistema de navegación interior basado en imágenes para personas con discapacidad visual. El sistema utiliza algoritmos de procesamiento de imágenes para extraer las regiones del piso de las imágenes capturadas desde un dispositivo portátil de visualización como GoogleGlass, que porta la persona. El algoritmo de detección de piso se probó con más de 200 imágenes capturadas desde pasillos interiores con diferentes condiciones de iluminación y logró una precisión de hasta el $81.8 \%$.

En Hungría, un grupo de estudiantes reportan haber desarrollado una aplicación móvil que utiliza tecnología de sistema de posicionamiento interior con Android y Bluetooth de baja energía (BLE) utilizando el método de trilateración (Noertjahyana, Wijayanto $\&$ 
OGEO: Sistema de navegación interior para la orientación y movilidad de personas con discapacidad visual

Andjarwirawan, 2017). Otro proyecto húngaro (Satan, 2018), mediante el uso del algoritmo de Dijkstra y dispositivos iBeacon, diseñó una aplicación para dispositivos móviles Android que permite determinar la ruta más corta entre un punto $A$ y un punto $B$. La aplicación no contiene un mapa del entorno, por lo que trabaja con imágenes que se actualizan a cada 5 metros y el usuario es guiado por unas flechas que se muestran en la pantalla. En una base de datos de SQLite se almacena la información necesaria del entorno: destinos, límites, salones, etc. Aunque la aplicación no está dirigida específicamente a una PcDV, como resulta obvio, posee ciertas características que permitirían una funcionalidad adecuada para que sea adaptada a una aplicación para PcDV para su navegación autónoma en interiores.

Otra situación que tiene que ser considerada es, el cómo desplegar los dispositivos sensores (balizas iBeacon) dentro de un edificio, los cuales deben interactuar con los teléfonos móviles para proporcionar la información necesaria para realizar la navegación. Así, para facilitar el análisis de distribución de dispositivos iBeacon en un determinado edifico, una técnica denominada IBeaconMap puede ser implementada con éxito (Cheraghi, Namboodiri \& Sinha, 2018). Esta técnica crea estructuras topológicas que representan un espacio interior, para usar con la navegación basada en balizas y que solo necesita los planos de los espacios interiores del edificio. IBeaconMap emplea una combinación de visión artificial y técnicas de aprendizaje automático para llegar al conjunto requerido de ubicaciones de balizas y un gráfico de conectividad ponderada (con orientaciones direccionales) para las necesidades de navegación posteriores. Para poder conocer los puntos de interés de un edificio y así ser registrado por los iBeacon, se analizan y procesan las imágenes o el documento del plano arquitectónico del edificio, por medio de un proceso de cuatro fases, descritas en Cheraghi et al. (2018). A continuación, se mencionan estas cuatro fases, con la finalidad de que se cuente con una idea básica de cómo se realiza este procedimiento. En la fase 1 , se identifica y se extrae el camino interior dentro del espacio respectivo de la planta y se identifican los puntos de interés. En la fase 2, se identifican todos los bloques de construcción dentro de la imagen del plano, esto es, puertas, escaleras, ascensores, etc., 
los cuales también pueden ser puntos de interés. En la fase 3, se genera un esqueleto de las rutas interiores utilizando los bloques de construcción y/o puntos de interés, teniendo en cuenta que las rutas posibles no pasen a través de las paredes, por ejemplo. Finalmente, en la fase 4 , se crea un gráfico de conectividad a través del esqueleto de rutas elaborado anteriormente, para generar rutas específicas que cuenten con la orientación requerida.

Otra aplicación que utiliza medios visuales para la navegación en interiores puede ser vista en Renuka, Yesodha, Perumal \& Dharanidahara (2018). En este trabajo se realiza el procesamiento de datos en la nube para estimar la ubicación de una persona que porta un teléfono móvil o el propio teléfono, si este ha sido extraviado. Dado que todo el proceso se realiza en la nube, un usuario solo a través de la aplicación móvil instalada identifica el iBeacon más cercano del dispositivo móvil extraviado, por ejemplo, y envía su latitud y longitud de este, al usuario dueño o a una estación de control para recuperar el dispositivo. Sin embargo, este desarrollo no está pensando para PcDV.
Otro trabajo interesante es el presentado en Cecilio, Duarte y Furtado (2015), en el cual se ha implementado un sistema para minimizar la cantidad de interacciones que una PcDV debe realizar para especificar los destinos o servicios deseados. El sistema determina la ubicación en exteriores a través del GPS y en interiores utilizando balizas de Bluetooth instaladas en el edificio. El usuario solicita a la aplicación la ubicación, la distancia al destino o los puntos de interés cercanos a él. Un sintetizador convierte el texto en instrucciones de voz, pero también acepta la búsqueda por voz. Esta aplicación hace uso del concepto de huella digital (fingerprinting en inglés), a través de un archivo $X M L$, que describe la localización en que se encuentra la persona con discapacidad visual y utiliza el algoritmo de Dijkstra para estimar la ruta más corta al destino deseado.

\section{METODOLOGÍA DE DESARROLLO DE APLICACIONES PARA PcDV}

Las recomendaciones para el diseño y desarrollo de sistemas de telecomunicaciones para el apoyo a las personas con discapacidad visual son presentadas por la Unión Internacional 
OGEO: Sistema de navegación interior para la orientación y movilidad de personas con discapacidad visual

de Telecomunicaciones (UIT) (en inglés,

International

Telecommunication Union - ITU) (ITU, 2018). En estas recomendaciones se explican una serie de criterios que deben cumplir los sistemas de navegación basados en audios para que sean utilizados por las PcDV. Estos sistemas deben ser inclusivos y adoptar una tecnología neutral, para el uso de toda la población en general. El usuario durante la navegación debe recibir información del entorno, puntos de interés, instrucciones de orientación, dirección, inicio de ruta, de dirección progresiva, además de las alertas. Es necesario que puntos como escalones, escaleras, elevadores, entradas, plataformas o estaciones de tren, sean contemplados en la información del entorno ofrecida al usuario, entre otros múltiples detalles que destaca la UIT en sus recomendaciones.

La organización sin fines de lucro Wayfindr (Wayfindr, 2018) desarrolla proyectos dirigidos a la creación de sistema tecnológicos para PcDV, basados en los criterios de la ITU-T F.921, en dónde se propone que dichos sistemas deben cumplir las siguientes características:
- Proporcionar información de posicionamiento de la PcDV.

- Proporcionar información sobre el entorno de en dónde está esta persona.

- Proporcionar información importante de diversos detalles que están sobre el punto de interés.

- Proporcionar las indicaciones, paso a paso, para navegar desde un punto $A$ un punto $B$.

El sistema debe contar con instrucciones para la navegación audible, además de un mapa e información del entorno. Wayfindr también establece que la funcionalidad que contienen las aplicaciones se debería basar en:

- Hacer uso de una alerta de notificación antes de una alerta o instrucción;

- Proporcionar previamente al usuario, por medio de instrucciones de audio, una determinada ruta que pretenda realizar (siempre que esta ruta proporcione una navegación);

- Permitir a un usuario reproducir un mensaje de audio;

- Habilitar la búsqueda por medio de dictado al usuario;

- Enviar una alerta de notificación al usuario indicando que la ruta ha 
sido completada y que ha llegado a su destino.

\section{DESARROLLO DE OGEO}

Ciertamente OGeo, más que una aplicación, es un sistema muy útil para proporcionar ayudas en el desplazamiento autónomo de PcDV en interiores de edificios. Esto se debe a que reúne las funcionalidades principales para que se lleve a cabo el desplazamiento. En este sistema se destaca la detección de balizas iBeacon para la navegación, la incorporación de puntos de interés por medios de la comparación de datos previamente almacenados en un servidor web y la modificación de estos puntos a medida que el usuario se desplaza. Además, otra característica destacable de este sistema es, ser una plataforma abierta para incorporar nuevas funcionalidades. Para ello, se pueden adaptar otros sensores e integrar nuevas técnicas de navegación compatibles con el sistema implementado.

\section{MODELO ARQUITECTÓNICO}

OGeo utiliza un modelo cliente/servidor. La PcDV utiliza un teléfono móvil que posee un paquete de aplicaciones para Android (.apk), propia de OGeo. La app mediante un módulo de programación está constantemente escaneando el ambiente en búsqueda de dispositivos iBeacon que estén emitiendo una señal alcanzable por el dispositivo móvil y que se encuentren registrados en su base de datos local.

Inicialmente, todos los datos son almacenados en una base de datos MySQL localizada en un servidor web, y posteriormente descargados al teléfono y almacenados en un base de datos SQLite, que brinda independencia de conexión. El modelo arquitectónico se observa en la figura 1.

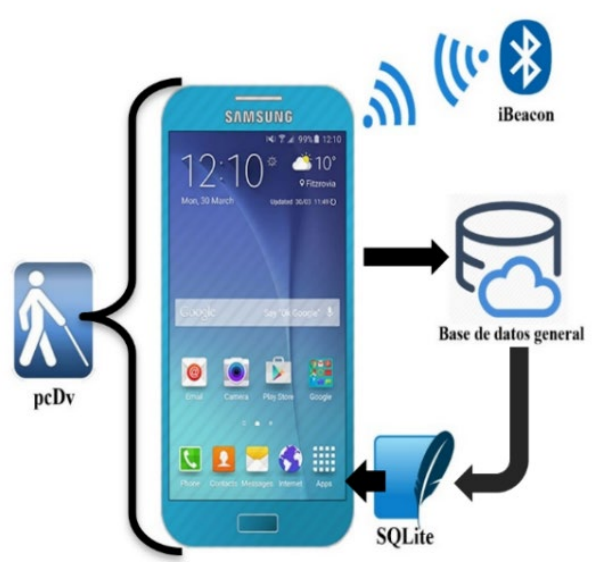

Figura 1. Arquitectura del Sistema OGeo.

Dentro

del modelo arquitectónico del sistema OGeo se encuentra la interfaz de usuario. Esta interfaz utiliza un sistema de clasificación de sitios, donde el lugar 
OGEO: Sistema de navegación interior para la orientación y movilidad de personas con discapacidad

que la PcDV va a recorrer es categorizado a fin de reducir la búsqueda de un destino. La pantalla de inicio es simple y las opciones de cada categoría se muestran como una lista que la PcDV puede recorrer fácilmente con el dedo, mientras escucha las diferentes opciones. La selección de una opción se lleva a cabo mediante dos toques rápidos de pantalla. Los usuarios de esta app son instruidos adecuadamente para que puedan utilizarla de manera eficiente.

\section{CONCEPTOS FUNDAMENTALES}

OGeo posee una serie de conceptos fundamentales que lo definen, presentados a continuación:

- Sitio. Un sitio es un edificio que posee un conjunto de dispositivos iBeacon que identifica diferentes lugares o destinos alcanzables por una PcDV. El sitio es la base de toda la información, ya que para cada sitio es almacenada una información única.

- Categoría. Una categoría es una clasificación de un sitio en áreas, como pueden ser oficinas, laboratorios, etc. De esta manera, se pueden agrupar los puntos de interés y reducir la información en la aplicación y la proporcionada al usuario para una facilidad en su utilización.

- Punto de Interés. Un punto de interés es un lugar específico dentro de un sitio, que puede corresponder a un baño, una oficina, un salón, escaleras, etc. Esta información es de suma importancia, ya que es la clave para favorecer el desplazamiento del usuario. Por lo tanto, la PcDV debe conocer la mayor cantidad de puntos de interés posibles que se encuentran en un edificio. Un punto de interés estaría representado por un dispositivo iBeacon, que contendrá la localización y la descripción del entorno en donde está instalado.

- Camino. El camino es la ruta establecida para desplazarse de un punto A hacia un punto $B$, previamente seleccionado por la PcDV.

- Intersección. La intersección es el lugar donde convergen dos o más posibles rutas. La decisión de escoger una de las rutas es establecida por la "ruta más corta" seleccionada por el sistema.

\section{DETECCIÓN DE UN PUNTO DE INTERÉS}


Para la detección de una baliza, que emite información por medio de Bluetooth, la cual representa un punto de interés, se utilizó la librería Altbeacon para Android. Esta librería permite discriminar las balizas de otros dispositivos Bluetooth que se encuentren en el entorno del dispositivo utilizado en el sistema OGeo.

Para la detección adecuada de las balizas por el dispositivo móvil, se considera la disposición o localización de ellas en el entorno, de la siguiente manera: las balizas se colocan a una altura de $2.1 \mathrm{~m}$, aproximadamente, mientras que, el usuario hace el recorrido con el teléfono a una altura aproximada de $1.3 \mathrm{~m}$ a $1.5 \mathrm{~m}$, entre su pecho y abdomen, sujetando el dispositivo frente a él, como se observa en la figura 2.

\section{DETERMINACIÓN DE LA RUTA MÁS CORTA}

El primer paso, antes de iniciar el desplazamiento, es establecer cuál es el camino más corto para llegar a un determinado destino. Este proceso se inicia en el momento que la PcDV selecciona un destino. Inmediatamente después, el algoritmo implementado en el sistema que utiliza el recurso del algoritmo de Dijkstra (Javaid, 2013), para encontrar la ruta más corta por medio de iteraciones ponderadas, determina la ruta que debe ser utilizada desde el punto origen al punto destino seleccionado. Esto es posible ya que la información por cada punto de interés está almacenada en una base de datos local en el dispositivo móvil, con lo cual se puede determinar el camino.

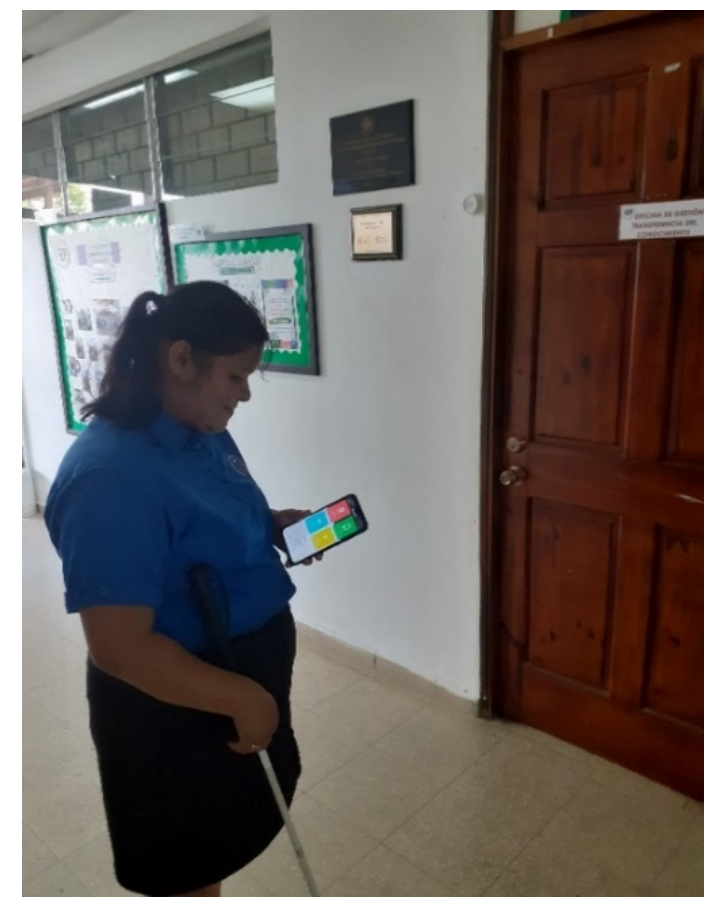

Figura 2. Imagen durante una prueba de desplazamiento en donde se percibe la colocación del dispositivo móvil.

\section{DESPLAZAMIENTO EN INTERSECCIONES}

El desplazamiento en intersecciones se representa como una variable de la forma $n-1$, donde $n$ representa el número de caminos 
OGEO: Sistema de navegación interior para la orientación y movilidad de personas con discapacidad visual

posibles en la intersección,

La instrucción consta de: exceptuando el camino de origen (-1).

Cada posible ruta desde la posición "origen", en la intersección, está relacionada a las diferentes direcciones que hay en una intersección, y de allí se indica hacia dónde debe continuar el desplazamiento. De esta forma, se logra notificar la dirección hacia la que debe dirigirse la PcDV. Las notificaciones en las intersecciones le indican a la PCDV la ruta a seguir dependiendo del origen de su desplazamiento. Esta información es muy importante y se debe notificar de manera correcta en los puntos de intersección, para evitar confusiones en la recepción del mensaje. A continuación, se analizan dos mensajes sonoros durante el desplazamiento:

(1) Ejemplo de mensaje sonoro: "Gire a la izquierda y continúe derecho".

La instrucción contiene los siguientes elementos: verbo ("gire"), delimitador direccional ("a la izquierda"), verbo ("continuar"), delimitador direccional ("derecho").

(2) Ejemplo de mensaje sonoro: "A pocos metros se encuentra una pequeña rampa y a continuación un teléfono público. Continúe despacio $"$. 
continuación, se describen brevemente las pruebas experimentales realizadas, que servirán para conocer el nivel de usabilidad del producto (Ferré, 2005) y modificarlo, dentro de los márgenes pertinentes:

- Prueba de proximidad. Esta prueba se realiza con el fin de detectar las balizas a una distancia de $1.0 \mathrm{~m}$ del usuario. Cada una de estas balizas representa un punto específico dentro del pasillo del edificio en donde se realizó la prueba. El resultado obtenido fue satisfactorio, incluyendo el cambio de codificación de los parámetros de detección de las balizas.

- Prueba de caminos. La PcDV selecciona un destino que se muestra en una lista en pantalla. Esta lista se comprueba con los valores esperados en la prueba unitaria sobre el desplazamiento a fin de corroborar la efectividad del algoritmo de Dijkstra.

- Prueba en intersecciones. Esta prueba permite demostrar si el módulo de orientación en intersecciones informa correctamente la dirección que debe seguir la PcDV en su desplazamiento.
- Prueba de arribo al destino. Esta prueba comunica oportunamente a la PcDV su llegada al destino seleccionado.

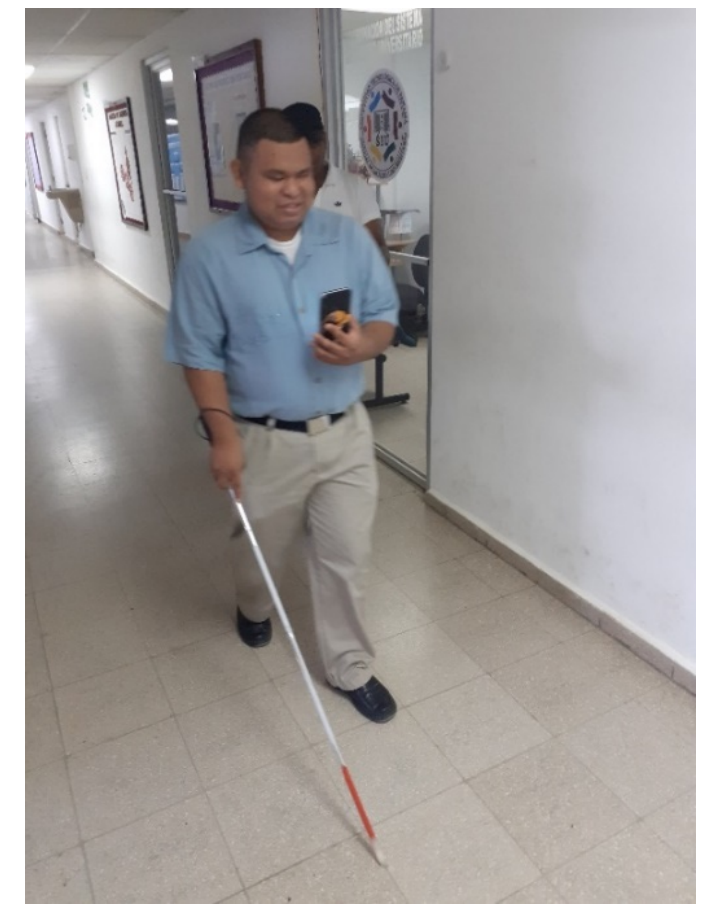

Figura 3. Captura de imagen de una PcDV en desplazamiento durante una de las pruebas de validación del sistema.

En la figura 3 se puede observar a una PcDV, que colabora con el proyecto de manera voluntaria, durante el recorrido en una de las pruebas realizadas.

\section{CONCLUSIONES}

El desarrollo de la app OGeo ha permitido la construcción de un sistema para ayudar en el desplazamiento autónomo a PcDV, que contribuirá a su inclusión social y educativa. La inversión económica para la realización 
OGEO: Sistema de navegación interior para la orientación y movilidad de personas con discapacidad visual

de este sistema es de bajo costo, si se REFERENCIAS compara, por ejemplo, con otros proyectos financiados en los EE.UU o en la UE (Cecílio, et al., 2015; Cheraghi, et al., 2018; Garcia, et al., 2015; Hicks, et al., 2013; Rivera-Rubio, et al., 2016; Wayfindr, 2018). Se han utilizado tecnologías accesibles, reduciendo la dependencia de la conectividad a Internet, y se han incorporado estándares internacionales encaminados al desarrollo de buenas prácticas dirigidas a las PcDV. El desarrollo de este proyecto supone un avance significativo para la independencia en cuanto a movilidad de las PcDV en el interior de edificios, como pueden ser los centros educativos.

\section{AGRADECIMIENTOS}

Este trabajo ha sido realizado dentro del marco del proyecto MOVIDIS-II financiado por la Secretaría Nacional de Ciencia, Tecnología e Innovación de Panamá (SENACYT) con el Contrato por Mérito NN 99-2018-4-FID17-031. Héctor Montes (Resolución 90-2017) agradece al Sistema Nacional de Investigación de Panamá (SNI) de la SENACYT por el apoyo que les proporciona en I+D a sus miembros.

Carballeda, G., Arcia, A., Pérez, R. y Montes, H. (2016). Aplicación móvil para el monitoreo de personas con discapacidad visual. Proc. Tecnología y accesibilidad. Aplicación de Tecnologías de la Información y Comunicaciones para mejorar la Accesibilidad, Cuenca, Ecuador, pp. 93-100. Recuperado de https://www.researchgate.net/publicati on/314007299_Aplicacion_Movil_para _el_Monitoreo_de_Personas_con_Dis capacidad_Visual.

Cecílio, J., Duarte, K., y Furtado, P. (2015). BlindeDroid: An Information Tracking System for Real-time Guiding of Blind People. Procedia Computer Science, 52, pp. 113-120. Recuperado de

https://cyberleninka.org/article/n/13343

94/viewer

Cheraghi, S. A., Namboodiri, V., \& Sinha, K. (2018). IBeaconMap: Automated Indoor Space Representation for Beacon-Based Wayfinding. Human-Computer Interaction. Recuperado de http://arxiv.org/abs/1802.05735 
De Benito, B. y Salinas, J. M. (2016). La investigación basada en diseño en Tecnología Educativa. RIITE. Revista Interuniversitaria de Investigación en Tecnología Educativa, 0, 44-59. doi: 10.6018/riite/2016/260631

Ferré, X. (2005). Marco de integración de la usabilidad en el proceso de desarrollo software (tesis doctoral). Universidad Politécnica de Madrid, España.

Garcia, G., \& Nahapetian, A. (2015, October 14). Demonstration paper: Wearable computing for image-based indoor navigation of the visually impaired. Proceedings - Wireless Health 2015, WH 2015. Recuperado de https://doi.org/10.1145/2811780.28119 59

Gelgon, M. \& Bouthemy, P. (2000). A region-level motion-based graph representation and labeling for tracking a spatial image partition. Pattern Recognition, 33(4), pp. 725-740.

Henríquez, A., Vejarano, R., Montes, H. (2017). OGeo: Aplicación para Ayuda en la Movilidad de Personas con Discapacidad Visual. In Proc. VIII Congreso Internacional sobre
Aplicación de Tecnologías de la Información y Comunicaciones Avanzadas (ATICA2017), 25-27, octubre, Medellín, Colombia, pp. 189196. Recuperado de http://www.cc.uah.es/Atica/documento s/LibroActasATICA2017.pdf

Hicks, S. L., Wilson, I., Muhammed, L., Worsfold, J., Downes, S. M., \& Kennard, C. (2013). A Depth-Based Head-Mounted Visual Display to Aid Navigation in Partially Sighted Individuals. PLoS ONE, vol. 8(7). doi: 10.1371/journal.pone.0067695

ITU. (2018). Audio-based indoor and outdoor network navigation system for persons with vision impairment. Series F: Non-Telephone Telecommunication Services. Accessibility and human factors. Recommendation ITU-T F.921. Recuperado de https://www.itu.int/rec/T-REC-F.921201808-I/en

Javaid, M. A. (2013). Understanding Dijkstra's Algorithm. SSRN Electronic Journal, 2340905, pp. 1-27. Recuperado de

http://dx.doi.org/10.2139/ssrn.2340905 
OGEO: Sistema de navegación interior para la orientación y movilidad de personas con discapacidad visual

López Pereda, P., y Borau Jordán, J. L. (2011). Diseño arquitectónico para todas las personas. Accesibilidad Universal y Diseño para Todos. Arquitectura y Urbanismo. Fundación ONCE y Fundación Arquitectura COAM, España, pp. 82-105. Recuperado de https://www.fundaciononce.es/sites/def ault/files/docs/Accesibilidad\%2520univ ersal\%2520y\%2520diseño\%2520para \%2520todos_1.pdf

Montes, H., Chang, I., Carballeda, G., Muñoz, J., García, A., Vejarano, R., Armada, M. (2016). MOVIDIS: first steps toward help the mobility of people with visual disability in Panama. Proc. RoboCity16 Open Conference on Future Trends in Robotics, Madrid, Spain, pp. 211-218. Recuperado de https://www.car.upm-csic.es/wpcontent/uploads/2016/06/Proceedings _RoboCity16_web_version_01.pdf

Montes, H., Chang, I., Carballeda, G., Muñoz, J., Garcia, A., Vejarano, R., Saez, Y. (2018). Design of a System to Support the Mobility of Visually Impaired People. Proc. Robotics transforming the future - CLAWAR 2018, Panamá, pp. 37-44. Recuperado de https://revistas.utp.ac.pa/index.php/me moutp/article/view/1874

Montes, H., Chang, I., Carballeda, G., Muñoz, J., Saez, Y., Vejarano, R. y Garcia, A. (2019). Conceptual design of technological systems for the mobility of visual impairment people in indoor buildings. Proc. 7th International Engineering, Sciences and Technology Conference (IESTEC), Panamá, pp. 16. [Online]. Recuperado de: https://ieeexplore.ieee.org/document/8 943749

Noertjahyana, A., Wijayanto, I. A., \& Andjarwirawan, J. (2017). Development of mobile indoor positioning system application using Android and bluetooth low energy with trilateration method. Proceedings - 2017 International Conference on Soft Computing, Intelligent System and Information Technology: Building Intelligence through IOT and Big Data, ICSIIT 2017. doi: 10.1109/ICSIIT.2017.64

ONCE. (2011). Desplazamiento por espacios interiores. Discapacidad visual y autonomía personal: enfoque práctico de la rehabilitación, 1a ed., España, pp. 516-531. Recuperado de http://riberdis.cedd.net/bitstream/handl 
Gómez, Rodríguez, Vejarano, Montes.

e/11181/3279/Discapacidad_visual_y_ autonomia_personal.pdf?sequence $=2$

Rana, S. (Octubre 2017). Direction of a Point from a Line Segment. GeeksforGeeks, A computer science portal for geeks [Blog]. Recuperado de https://www.geeksforgeeks.org/directio n-point-line-segment/

Renuka, G., Yesodha, M., Perumal, S., Dharanidahara, K. (2018). Design of BLE based location tracking using beacon. International Journal of Pure and Applied Mathematics, 119(12), pp. 197-201. Recuperado de https://acadpubl.eu/hub/2018-11912/articles/7/1587.pdf

Rivera-Rubio, J., Arulkumaran, K., Rishi, H., Alexiou, I., \& Bharath, A. A. (2016). An assistive haptic interface for appearance-based indoor navigation. Computer Vision and Image Understanding, vol (146), pp. 126-145. doi: 10.1016/j.cviu.2016.02.014

Romero, D., y Rodríguez, F. (2018). Diseño de un Sistema Portátil para la Detección de Obstáculos por medio de Kinect, como Apoyo a Personas con Limitaciones Visuales. Trabajo de Grado. Universidad Distrital Francisco
José de Caldas, Bogotá, Colombia. Recuperado de http://repository.udistrital.edu.co/handl e/11349/14039

Satan, A. (2018). Bluetooth-based indoor navigation mobile system. Proceedings of the 2018 19th International Carpathian Control Conference, ICCC 2018. Recuperado de

https://doi.org/10.1109/CarpathianCC. 2018.8399651

Wayfindr (1 August 2018). Understanding Audio Wayfinding and Audio-Based Navigation. Wayfindr nonprofit organisation. Recuperado de https://www.wayfindr.net/wayfindrelearning 